\title{
Escalas de avaliação da qualidade de vida em pacientes brasileiros após acidente vascular encefálico
}

\author{
Quality of life scales for Brazilian stroke patients
}

\section{Marcos Roberto de Oliveira ${ }^{1}$, Marco Orsini ${ }^{2}$}

\begin{abstract}
RESUMO
Introdução. A qualidade de vida após o acidente vascular encefálico (AVE) e os métodos de mensuração são de grande importância. O objetivo do estudo é apresentar as escalas que são utilizadas para avaliação de qualidade de vida de pacientes adultos após $A V E$, analisando a validade e uso dos instrumentos na população brasileira. Método. Descrever as escalas de qualidade de vida: Instrumento de Avaliação de Qualidade de Vida da Organização Mundial de Saúde (WHOQOL-100 e Bref), Perfil de Saúde de Nottingham (PSN), Formulário Abreviado da Avaliação de Saúde 36 (SF-36) e a Escala Específica de Qualidade de Vida no AVE (SS-Qol). Foi realizado levantamento nas fontes eletrônicas da MEDLINE, PUBMED, LILACS e Scielo. Foram incluídos estudos referentes ao período entre 1990-2007. Conclusão. Os artigos encontrados mostraram que as escalas estudadas são as mais adequadas para aplicação na população brasileira, com boa consistência interna, validade e confiabilidade teste-reteste. Apesar de serem escalas reconhecidas internacionalmente, ainda são necessários mais estudos clínicos aplicando-as nos indivíduos após AVE no Brasil, para melhor assegurar as suas propriedades psicométricas, aplicabilidade, melhor forma de administração e as possíveis interferências inter-examinadores.
\end{abstract}

Unitermos. Qualidade de Vida, Acidente Vascular Encefálico, Escalas.

Citation: Oliveira MR, Orsini M. Escalas de avaliação da qualidade de vida em pacientes brasileiros após acidente vascular encefálico.

\section{SUMMARY}

Introduction. The quality of life in stroke patients and how to measure it are very important. This study aims to present scales that are used for evaluating quality of life in stroke adult-patients, considering the validity and application of its instruments in Brazil. Method. The quality of life scales described are The World Health Organization Quality of Life Assessment Instrument (WHOQOL-100 and Bref), Nottingham Health Profile (NHP), Medical Outcomes Study 36 Item Short Form Health Survey (SF-36) and, Stroke-Specific Quality of Life Measure (SS-QOL). References were identified by electronic searches of MEDLINE, PUBMED, LILACS, and Scielo. We included papers published both 1990-2007. Conclusion. The found papers showed that the studied scales were the most useful for application in Brazil, with good internal consistency, validity, and reliability test-retest. Therefore the scales were known in world, there still is a necessity for more studies about its application in Brazilian stroke patients, psychometrics features, administration form, and internal validity.

Keywords. Quality of life, Stroke, Scales.

Citation: Oliveira MR, Orsini M. Quality of life scales for Brazilian stroke patients.
Trabalho realizado na Universidade Gama Filho - UGF.

1. Fisioterapeuta da Rede SARAH de Hospitais de Reabilitação, Especialista em Fisioterapia Neurofuncional pela Universidade Gama Filho-UGF.

2. Fisioterapeuta, Professor Titular de Reabilitação Neurológica e Doutorando em Neurociências - HUAP - Universidade Federal Fluminense- UFF.
Endereço para correspondência: Marcos Roberto de Oliveira R. Rodolpho Coelho Cavalcante, 115/1003 41750-166 Salvador, BA E-mail: marcos.oliveira@sarah.br / orsini@predialnet.com.br

Recebido em: 07/11/07 Revisado em: 08/11/07 a 16/03/08 Aceito em: $17 / 03 / 08$ Conflito de interesses: não 


\section{INTRODUÇÃo}

O acidente vascular encefálico (AVE) é um dos maiores problemas de saúde pública nos últimos anos, sendo considerada uma das doenças mais incapacitantes. Dentre as incapacidades, podemos citar os prejuízos das funções motoras, limitação das atividades de vida diária, possível presença de déficits cognitivos e de linguagem, depressão e restrições para o convívio social, podendo assim, influenciar na qualidade de vida ${ }^{1-3}$.

Freqüentemente, estudos epidemiológicos focalizam a mortalidade e recorrência, mas não os problemas relacionados à qualidade de vida ${ }^{1,4}$. Em recente estudo, encontrou-se uma incidência total de casos de AVE na população da América Latina e Caribe de 140/100.000 habitantes, e a prevalência de sobreviventes de AVE, com recuperação incompleta, estimada em 460/100.0005. Dados de estudos nacionais demonstram incidência variando entre $156 / 100.000^{4}$ e $168 / 100.000^{6}$. A mortalidade no primeiro ano é estimada em $15 \%$ e $25 \%$, recorrência entre $5 \%$ e $14 \%$, incapacidade parcial ou completa entre $24 \%$ e $54 \% 2$.

O termo qualidade de vida foi definido em 1994 pelo grupo de qualidade de vida da Organização Mundial da Saúde (OMS), como sendo "a percepção do indivíduo de sua posição na vida no contexto da cultura e sistema de valores no qual ele vive e em relação a seus objetivos, expectativas, padrões e seus interesses"1,2,7,8. Dessa forma, 0 conceito de qualidade de vida tem sido interpretado em diversas perspectivas, incluindo o bem-estar físico, psicológico e espiritual, além de aspectos sociais, econômicos e políticos ${ }^{3,9}$.

Nos últimos 20 anos, a medida de qualidade de vida vem surgindo no meio científico como instrumento importante para a investigação e a avaliação de pacientes, principalmente os com enfermidades crônicas ${ }^{7}$. Os resultados auxiliam, inclusive, como um dos critérios para a liberação do uso de novas drogas em vários países ${ }^{7,10}$ e para acompanhamento de pacientes que participaram de programa de reabilitação ${ }^{3}$.

Na Europa e nos Estados Unidos, a avaliação de qualidade de vida é extensamente aplicada para os pacientes com AVE ${ }^{1-3,11,12}$, com alguns estudos específicos para este tema no Brasil ${ }^{10,13,14}$.

Os instrumentos utilizados para a avaliação de qualidade de vida foram desenvolvidos para as maisdiversasfinalidadeseindicações, sendorealizadas através de escalas genéricas do estado de saúde e escalas específicas a uma determinada doença ${ }^{15}$.

O objetivo do presente estudo de revisão da literatura é apresentar os instrumentos que são utilizados para avaliação de qualidade de vida de pacientes adultos após $\mathrm{AVE}$, referenciados na literatura mundial, discutindo a validade e aplicação desses instrumentos na população brasileira.

\section{MÉTODO}

Foram pesquisados artigos nas fontes eletrônicas da MEDLINE, PUBMED, LILACS e Scielo, referentes ao período entre 1990-2007. As publicações foram do tipo revisões, estudos coorte, estudos transversais e estudos clínicos. Os termos utilizados no levantamento foram: "Stroke", "Quality of Life", "Measurement", "Disability", "Scale", e os referentes termos em português (Acidente Vascular Encefálico, Qualidade de Vida, Mensuração, Incapacidade e Escalas) para busca em fontes nacionais.

\section{RESULTADOS}

A adoção da terminologia qualidade de vida resulta de um processo histórico cujas primeiras tentativas conceituais surgiram a partir de 384 a.C., quando Aristóteles se referia à associação entre felicidade e bem-estar ${ }^{1}$. Já naquele período, as diferenças interpessoais (variação entre pessoas distintas) e intrapessoal (variação de acordo com situações de vida específicas), em relação à satisfação com a vida, eram consideradas essenciais para a compreensão do bem-estar ${ }^{1,15}$.

A organização mundial de saúde (OMS) definiu saúde em 1948 como "um completo bem-estar físico, psíquico e social e não meramente ausência de doença" ${ }^{\prime 16}$. Apesar da definição ocasionar uma amplitude de aspectos, as avaliações até então realizadas consideravam apenas fatores de morbidade e mortalidade, deixando uma lacuna entre a definição conceitual de saúde e suas medidas². Posteriormente, o termo qualidade de vida foi definida em 1994 pelo grupo de qualidade de vida da Organização Mundial da Saúde (OMS) levando em consideração a percepção do indivíduo, cultu- 
ra, sistemas de valores, objetivos, expectativas, padrões e interesses ${ }^{1,3,7}$.

O termo qualidade de vida relacionada à saúde (do inglês, Health-related quality of life), utilizado, atualmente, é um subconjunto mais amplo, e refere-se à função do paciente em perceber sua performance em quatro dimensões: 1) física, 2) funcional, 3) psicológica e 4) social ${ }^{3,9,17}$. Somandose aos aspectos físico, mental e social, existem novas considerações em relação à percepção pessoal, crenças pessoais, religiosidade e espiritualidade que necessitam serem incorporadas ao conceito relacionado à saúde ${ }^{3,11}$. Esses domínios refletem, em parte, os itens da nova Classificação Internacional de Impairments, Activity e Participation da Organização Mundial de Saúde (OMS) ${ }^{18}$.

Os objetivos da mensuração da qualidade de vida são: descrever a qualidade de vida de uma população e sua mudança ao longo do tempo; identificar os preditores para a qualidade de vida em uma populaçãoeanalisarocusto-benefíciodeumadeterminada intervenção $0^{8,11,15}$.

Instrumentos para avaliação da qualidade de vida

Os instrumentos de avaliação de qualidade de vida foram desenvolvidos para as mais diversas finalidades, podendo ser através de escalas genéricas do estado de saúde e escalas específicas relacionadas à doença².

As escalas genéricas podem ser usadas na população geral para avaliar uma larga faixa de domínios e permitir comparações entre diferentes condições ou doenças. Exemplos de escalas genéricas existem: Escala de Qualidade de Vida utilizada na Europa (do inglês, Euroqol) ${ }^{15,19}$, Escala de Qualidade do Bem-estar (QWBS - do inglês, Quality of Well-Being Scale) ${ }^{15,19}$, Índice de Qualidade de Vida Ferrans \& Powers ${ }^{15,19}$, Perfil do Impacto da Doença (SIP - do inglês, Sickness Impact Profile) $)^{15,19}$, Instrumento de Avaliação de Qualidade de Vida da Organização Mundial de Saúde (WHOQOL - do inglês, The World Health Organization Quality of Life Assessment Instrument) ${ }^{7}$, Perfil de Saúde de Nottingham (NHP - do inglês, Nottingham Health

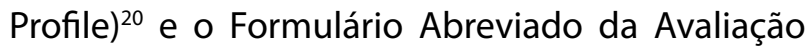
de Saúde 36 (SF-36 - do inglês, Medical Outcomes Study 36 - Item Short Form Health Survey) ${ }^{21,22}$. Destas escalas apresentadas, as que apresentam estudos de tradução e adaptação cultural para a população brasileira são: WHOQOL-100 7 e WHOQOL-Bref', SF-36 ${ }^{22}$ e NHP ${ }^{20}$.

As escalas específicas focam em problemas específicos de uma determinada doença e são indicadas para um ensaio no qual uma intervenção específica está sendo avaliada. Exemplos de escalas específicas para o AVE: Escala Específica de Qualidade de Vida no AVE (SS-QOL - do inglês, Strokespecific of Quality of Life) ${ }^{10,23}$, Escala de Qualidade de Vida de Niemi ${ }^{15,24}$, Entrevista de Satisfação de Vida de Viitanen ${ }^{15,24}$, Instrumento de Qualidade de Vida para AVE Hemorrágico ${ }^{15,24}$, Escala do Impacto do AVE versão 3.0 (SIS - do inglês, Stroke Impact Scale) ${ }^{15,24}$, e a Escala de Qualidade de Vida em pacientes com AVE e Afasia (SAQOL-39 - do inglês, Stroke and Aphasia Quality of Life Scale) ${ }^{25}$. Dentre as escalas específicas apresentadas, apenas a SSQOL possui estudo de tradução e adaptação cultural para a população brasileira ${ }^{10}$.

Devido o objetivo dessa revisão ser a apresentação da validade e aplicação desses instrumentos na população brasileira, discutiremos apenas as escalas WHOQOL-100, WHOQOL-Bref, SF-36, NHP e a SS-QOL.

Escalas genéricas para avaliação da qualidade de vida

Formulário Abreviado de Avaliação de Saúde 36 (SF-36)

A SF-36 é um instrumento genérico de avaliação da qualidade de vida, de fácil aplicação e compreensão. É um questionário multidimencional formado por 36 itens, englobados em 8 escalas ou componentes. Suas medidas de qualidade de vida são consideradas "padrão-ouro" nos estudos relacionados à saúde ${ }^{3,17,26}$, além do uso no desenvolvimento e testes de outros instrumentos ${ }^{15,17}$. Na população com AVE, a SF-36 já foi validada e é extensamente utilizada $a^{3,11,17,19}$.

Nototal oito conceitos de saúde são avaliados pela SF-36 que são eles: a funcionalidade física, limitações em atividades usuais devidoà doença, dor corporal, percepções gerais da saúde, vitalidade/ energia, limitações em atividades sociais devido à doença, limitações em atividades usuais devido aos problemas emocionais e saúde mental geral ${ }^{26}$.

Quanto às qualidades psicométricas, os estudos descrevem que a SF-36 é sensitiva às mudanças 
ao longo do tempo na qualidade de vida relacionada à saúde de pessoas com incapacidades, assim como reúne validade e confiabilidade na sua utilização ${ }^{11,19,26}$. No estudo de tradução e adaptação cultural para a população brasileira, foram avaliados 50 pacientes com diagnóstico de Artrite Reumatóide, concluindo-se que a SF-36 é uma escala valida para ascondiçõessocioeconômicaseculturais dapopulação brasileira, assim como a sua reprodutibilidade, apresentandocomoinstrumentoútildeavaliaçãode qualidade de vida no Brasil ${ }^{22}$ (Tabela 1).

No meado da década de 90, uma versão abreviada da escala, conhecida como SF-12, foi desenvolvida ${ }^{27}$. Os componentes resumidos das escalas foram os físicos e mentais, mantendo a mesma acurácia em relação à escala SF-36 original. Alguns estudos mostram que os componentes físicos e mentais da escala SF-36 são replicados pela escala SF-12 ${ }^{21}$. A SF-12 pode ser recomendada como apropriada no substituto da SF-36 em pacientes com AVE, porém mais estudos são necessários com a aplicação desta forma reduzida nesse grupo de pacientes. Até o presente momento, não foram encontrados estudos brasileiros que utilizassem a escala SF-12 em pacientes após AVE.

Instrumento de Avaliação de Qualidade de Vida da Organização Mundial de Saúde (WHOQOL)

A busca por um instrumento que avaliasse qualidade de vida dentro de uma perspectiva essencialmente internacional fez com que a Organização Mundial da Saúde (OMS), desenvolvesse um projeto colaborativo multicêntrico. Quinze centros estiveram envolvidos nesta etapa: Melbourne (Austrália), Zagreb (Croácia), Paris (França), Nova Delhi (Índia), Madras (Índia), Beer-Sheeva (Israel), Tóquio (Japão), Tilburg (Holanda), Panamá (Panamá), São Petersburgo (Rússia), Barcelona (Espanha), Bangkok (Tailândia), Bath (Reino Unido), Seattle (EUA) e Harare (Zimbabwe). O resultado deste projeto foi à elaboração do WHOQOL-100, um instrumento de avaliação de qualidade de vida, composto por 100 itens, avaliando seis domínios da qualidade de vida relacionada à saúde: físico, psicológico, nível de independência, relações sociais, aspectos do meio ambiente e espiritualidade/religião/crenças pessoais $^{28}$. Quatro questões são formuladas para cada uma das 24 facetas, compondo 96 das 100 questões, e outras quatro questões são dedicadas a aspectos subjetivos da qualidade de vida. Todas as respostas são compostas por escala graduada tipo Likert, com escores que variam de um a cinco pontos $^{29}$. Devido ao número elevado de questões, o tempo estimado de aplicação é em torno de 60 a 90 minutos, podendo ser uma escala de reduzida aplicabilidade prática na utilização diária.

Frente à necessidade de instrumentos que demandem tempo menor para preenchimento e que mantenham a qualidade das características psicométricas do WHOQOL-100, foi desenvolvida uma versão abreviada da escala, denominada Instrumento Abreviado de Avaliação de Qualidade de Vida da Organização Mundial da Saúde (WHOQOL-Bref) $)^{7}$. A WHOQOL-Bref é composta de 26 questões divididas em 4 domínios: físico, psicológico, relações sociais e meio ambiente (Tabela 1). Alguns estudos demonstram a sua utilização em pacientes após AVE ${ }^{12,30}$.

Os resultados do processo de validação da versão em português do WHOQOL-Bref', realizados nos mesmos moldes do processo desencadeado para a validação do WHOQOL-100 no Brasil e nos demais centros de pesquisa ${ }^{28,29}$, apontaram um bom desempenho psicométrico com características satisfatórias de consistência interna, validade discriminante, validade de critério, validade simultânea e confiabilidade teste-reteste. Desta forma, o modelo completo e abreviado da WHOQOL são alternativas úteis para serem utilizadas em estudos que se propõe a avaliar qualidade de vida no Brasil ${ }^{7,29}$.

São necessários mais estudos brasileiros para a análise das correlações dos dados psicométricose sua aplicabilidade na população brasileira com AVE, apesar de ser uma escala confiável, válida e aplicável em estudos já realizados internacionalmente ${ }^{12,30}$.

Perfil de Saúde de Nottingham (PSN)

O Perfil de Saúde de Nottingham é um instrumento genérico de avaliação de qualidade de vida, desenvolvido originalmente para avaliar a qualidade de vida em pacientes portadores de doenças crônicas ${ }^{20}$. O Perfil de Saúde de Nottingham é baseado na Escala do Impacto do AVE (SIS - do inglês, Stroke Impact Scale), sendo um instrumento mais utilizado na Europa do que nos Estados Unidos da América e Brasil, onde as pesquisas utilizam mais a SF-36 ${ }^{13,15}$. 
Tabela 1. Características dos instrumentos genéricos para avaliação no AVE.

\begin{tabular}{|c|c|c|c|c|c|}
\hline Instrumento & $\mathrm{N}^{\circ}$ de itens & Domínios & $\begin{array}{c}\text { Forma de adminis- } \\
\text { tração }\end{array}$ & Tempo de aplicação & Dados psicométricos \\
\hline SF- $36^{20}$ & 36 & $\begin{array}{l}\text { Funcionalidade física, limitações } \\
\text { em atividades usuais devido a } \\
\text { doença, dor corporal, percep- } \\
\text { ções gerais da saúde, vitalidade/ } \\
\text { enegia,limitações em atividades } \\
\text { sociais, limitações em atividades } \\
\text { usuais devido problemas emo- } \\
\text { cionais, saúde mental geral }\end{array}$ & $\begin{array}{l}\text { Entrevista, auto-res- } \\
\text { pondido (paciente e } \\
\text { proxy) }\end{array}$ & 5-10 minutos & $\begin{array}{l}\text { Confiabilidade, validade e } \\
\text { responsividade a mudanças } \\
\text { extensamente testados }\end{array}$ \\
\hline $\begin{array}{l}\text { Perfil de saúde de } \\
\text { Nottingham }^{15}\end{array}$ & 38 & $\begin{array}{l}1^{\circ} \text { parte: mobilidade física, } \\
\text { energia, dor, sono, reações } \\
\text { emocionais, isolação social. } 2^{\circ} \\
\text { parte:trabalho,relacionamentos } \\
\text { e atividades sociais, atividades } \\
\text { em casa, atividade sexual. }\end{array}$ & $\begin{array}{l}\text { Entrevista, auto-res- } \\
\text { pondido (paciente) }\end{array}$ & 5-15 minutos & $\begin{array}{l}\text { Confiabilidade, validade e } \\
\text { responsividade a mudanças }\end{array}$ \\
\hline WHOQOL-10021 & 100 & $\begin{array}{l}\text { Domínio físico; domínio psico- } \\
\text { lógico; nível de independência; } \\
\text { relações sociais; meio ambiente; } \\
\text { aspectos espirituais/crenças } \\
\text { pessoais/religião }\end{array}$ & $\begin{array}{l}\text { Auto-administrado, } \\
\text { assistidopeloentrevis- } \\
\text { tador e administrado } \\
\text { pelo entrevistador }\end{array}$ & 60 a 90 minutos & $\begin{array}{l}\text { Boa consistência interna, vali- } \\
\text { dade discriminante, validade } \\
\text { concorrente, validade de } \\
\text { conteúdo e confiabilidade } \\
\text { teste-reteste }\end{array}$ \\
\hline WHOQOL-Bref ${ }^{4}$ & 26 & $\begin{array}{l}\text { Domínio físico; domínio psi- } \\
\text { cológico; relações sociais; meio } \\
\text { ambiente }\end{array}$ & $\begin{array}{l}\text { Auto-administrado, } \\
\text { assistidopeloentrevis- } \\
\text { tador e administrado } \\
\text { pelo entrevistador }\end{array}$ & Não relatado & $\begin{array}{l}\text { Boa consistência interna, vali- } \\
\text { dade discriminante, validade } \\
\text { concorrente, validade de } \\
\text { conteúdo e confiabilidade } \\
\text { teste-reteste }\end{array}$ \\
\hline
\end{tabular}

Trata-se de um questionário auto-administrado, constituído de 38 itens, baseados na classificação de incapacidade descrita pela Organização Mundial da Saúde (OMS), com respostas no formato $\operatorname{sim} /$ não. Os itens estão organizados em seis categorias que englobam nível de energia, dor, reações emocionais, sono, interação social e habilidades físicas. Cada resposta positiva corresponde a um escore de um (1) e cada resposta negativa corresponde a um escore zero (0), perfazendo uma pontuação máxima de 38 (ver Tabela 1). Utilizando uma linguagem de fácil interpretação, o NHP fornece uma medida simples da saúde física, social e emocional do indivíduo, sendo considerado clinicamente válido para distinguir pacientes com diferentes níveis de disfunção e para detectar alterações importantes no quadro de saúde do paciente ao longo do tempo ${ }^{20}$.

No entanto, o instrumento apresenta problemas com relação à validade clínica, pois a escala é simples e não mede todo os domínios da qualidade de vida. Além disso, o questionário discrimina pouco o paciente, já que divide os indivíduos em apenas dois níveis de habilidade. Isso indica que ele poderia ser mais útil quando aplicado em indivíduos mais debilitados. Para sua aplicação em indivíduos mais independentes funcionalmente, é recomendada a revisão da escala e a inclusão de itens mais complexos ${ }^{20}$.
Portanto, apesar de ser um perfil simples e de fácil utilização, é fundamental a associação desse questionáriocomumaavaliaçãofuncionaleumaentrevista semi-estruturada, tornando as informações coletadas mais úteis clinicamente ${ }^{13,15,20}$.

\section{Escalas específicas para avaliação da qualidade de vida no AVE}

\section{Escala Específica de Qualidade de Vida no AVE (SS-QOL)}

Williams e cols. desenvolveram o primeiro instrumento específico de avaliação da qualidade de vida em pacientes após AVE, sendo baseado em entrevistas que seguiram um padrão psicométri$\mathrm{CO}^{23}$. Para a criação dos domínios e itens, foram realizadas entrevistas com pacientes após AVE do tipo isquêmico para identificar os domínios que comumente afetam a relação saúde-qualidade de vida destes pacientes. Os itens foram gerados a partir dessas respostas, por meio de revisões de outros instrumentos para avaliação do AVE e da relação saúde-qualidade de vida ${ }^{10,23}$.

A escala possui 49 itens em 12 domínios, e para cada item existem escores de até 5 pontos 10 (Tabela 2).

Recentes artigos publicados sobre a escala, concluem que a SS-QOL é uma medida válida e confiável da qualidade de vida no AVE e possui 
Tabela 2. Características do instrumento específico para avaliação no AVE.

\begin{tabular}{|c|c|c|c|c|c|}
\hline Instrumento & $\mathrm{N}^{\circ}$ de ítens & Domínios & Forma de administração & Tempo de aplicação & Dados psicométricos \\
\hline SS-QOL 5 & 49 & $\begin{array}{l}\text { Energia, papel na família, linguagem, } \\
\text { humor, personalidade, auto-cuida- } \\
\text { do, papel social, pensamento, visão, } \\
\text { função do membro superior, trabalho } \\
\text { e produtividade, } 1 \text { questão para avaliar } \\
\text { a qualidade de vida global antes e após } \\
\text { AVE }\end{array}$ & $\begin{array}{l}\text { Entrevista ou auto-res- } \\
\text { pondido }\end{array}$ & 20 minutos & $\begin{array}{l}\text { Confiabilidade, } \\
\text { validade e moderada } \\
\text { responsividade a } \\
\text { mudanças }\end{array}$ \\
\hline
\end{tabular}

moderada responsividade às mudanças ${ }^{10,31,32}$. Em sua versão inglesa, a escala SS-QOL apresentou resultados satisfatórios no que diz respeito à confiabilidade, responsividade e validade construtiva. $\mathrm{O}$ instrumento mostrou-se simples na aplicação e na compreensão dos itens, bem como na interpretação dos resultados obtidos ${ }^{23}$.

Ewert et al. recomendam a utilização da escala em incapacidade de leve a moderada em indivíduos com seqüela de AVE, e que após a avaliação dos pacientes com AVE do tipo isquêmico e hemorrágico no seu estudo, as características dos dois grupos (tipo isquêmico e tipo hemorrágico) podem influenciar nos resultados obtidos devido às características específicas dessas populações (como idade, severidade da incapacidade e participação laboral e social) ${ }^{31}$. Este fato pode limitar a generalização dos resultados.

Quando comparado com outros instrumentos genéricos de medida da qualidade de vida, a SS-QOL demonstrou maior cobertura das funções tipicamente afetadas pelo AVE ${ }^{10,14,24,31}$. Os instrumentos genéricos, como a SF-36 e o WHOQOL, não apresentam questionamentos sobre a linguagem, função das mãos, cognição e visão, itens contemplados pela SS-QOL.

Apenas a SS-QOL, dentre as escalas específicas existentes, possui estudo de traduçãoe adaptação cultural para a população brasileira ${ }^{10}$. No estudo brasileiro para tradução e adaptação cultural, foram avaliados pacientes com AVE isquêmicos, concluindo-se que a escala possui característica simples de aplicação e na compreensão dos itens, bem como na interpretação dos resultados. Dos Santos conclui também que o instrumento SS-QOLéadaptado culturalmente para a língua portuguesa falada no Brasil para a avaliação da qualidade de vida após AVE, mas necessita ainda de mais estudos clínicos com a sua utilização no Brasil, assegurandoas suas propriedades psicométricas, além dos já existentes ${ }^{10,14}$.
Variáveis que influenciam a qualidade de vida após AVE

A mensuração da qualidade de vida engloba uma ampla variedade de domínios da vida nos aspectos relativos ao bem-estar físico, psicológico e social. Aspectos como o ganho funcional que se relaciona ao desempenho nas atividades de vida diária, são os resultados mais avaliados na literatura após AVE7. Mas aspectos psicossociais devem ser analisados para que tenhamos uma visão mais abrangente da qualidade de vida relacionada à saúde.

Os fatores que mais se relacionaram com a qualidade de vida foram o desempenho funcional nas atividades de vida diária, depressão $0^{33}$ equestões psicossociais como suporte social, relacionamentos sociais do paciente, participação em atividades de lazer e retorno ao trabalho, ${ }^{2,11}$. Melhores níveis de suporte social e familiar tem sido relacionados a melhores resultados ${ }^{33-35}$.

O nível de independência funcional ou recuperação motora, apesar de sua relação com a qualidade de vida, não podem ser considerados em separado dos outros aspectos ${ }^{15,33,35}$.

Os resultados dos diversos estudos realizados sobre qualidade de vida, tanto com escalas genéricas quanto com as escalas específicas, demonstram a presença de depressão, disfunção sexual, agravamento da incapacidade, rede social insuficiente, afasias graves e os estados cognitivos alterados, como fatores que contribuem com a pobre percepção da qualidade de vida em pacientes com seqüela de $\mathrm{AVE}^{2,21,24}$. Entretanto, a adoção de estratégias adequadas de suporte para pacientes com seqüela de $A V E$, na sua reintegração a comunidade, a partir do conhecimento de quais fatores preditivos da qualidade de vida relacionada à saúde estão mais afetados, contribuem para a melhora da percepção de qualidade de vida ${ }^{21}$, além de auxiliar na adoção de medidas específicas e direcionadas a essa população ${ }^{11-13}$. 


\section{CONCLUSÃO}

A utilização da avaliação da qualidade de vida relacionada à saúde, em indivíduos com seqüela de $A V E$, forneceria um perfil mais global das condições funcionais, psicossociais do paciente e de suas expectativas em relação á vida, direcionando assim a reabilitação a objetivos mais específicos, que contribuiriam para uma maior humanização e efetividade do processo de reabilitação e para uma melhor percepção da qualidade de vida pelos pacientes.

A partir dos estudos levantados sobre as escalas de qualidade de vida mais adequadas para aplicação na população brasileira, com boa consistência interna, validade discriminante, validade de critério, validade simultânea e confiabilidade teste-reteste, são recomendadas todas as escalas apresentadas: SF-36, WHOQOL-100, WHOQOLBref, Perfil de Saúde de Nottingham e a SS-QOL.

Todas apresentam características de fácil aplicação, exceto a WHOQOL-100 que devido ao

\section{REFERÊNCIAS}

1.Fayer PM, Machin D. Quality of life - assessment, analysis and interpretation. Oxford: Wiley, 2000, 416p.

2.Carod-Artal J, Egido JA, González JL, Varela de Seijas E. Quality of life among stroke survivors evaluated 1 year after stroke: experience of a stroke unit. Stroke 2000;31:2995-3000.

3.Hopman WM, Verner J. Quality of life during and after inpatient stroke rehabilitation. Stroke 2003;34:801-5.

4.Cabral NL, Longo AL, Moro CHM, Kiss HC. Epidemiologia dos acidentes cerebrovasculares em Joinville, Brasil. Arq Neuropsiquiatr 1997;55:357-63.

5.Lavados PM, Hennis AJ, Fernandes JG, Medina MT, Legetic B, Hoppe $A$, et al. Stroke epidemiology, prevention, and management strategies at a regional level: Latin America and the Caribbean. Lancet Neurol 2007;6:362-72.

6.Lessa I, Bastos CAG. Epidemiologia dos acidentes vasculares encefálicos na cidade de Salvador, Bahia, Brasil. Bol Ofic San Panam 1984;96:404-12.

7.Fleck MPA, Louzada S, Xavier M, Chachamovich E, Vieira G, Santos $L$, et al. Aplicação da versão em português do instrumento abreviado de avaliação da qualidade de vida "WHOQOL-BREF". Rev Saúde Pública 2000;34:178-83.

8.Studenski S, Duncan PW, Perera S, Reker D, Lai SM, Richards L. Daily functioning and quality of life in a randomized controlled trial of therapeutic exercise for subacute stroke survivors. Stroke 2005;36:1764-79.

9.Hsiung PC, Fang CT, Chang YY, Chen MY, Wang JD. Comparison of WHOQOL-BREF and SF-36 in patients with HIV infection. Qual Life Res 2005;14:141-50. número elevado de questões e domínios avaliados, proporciona um tempo maior para a sua utilização.

Entre as escalas genéricas, a escala SF-36 é reconhecida como "padrão-ouro" para avaliação da qualidade de vida relaciona a saúde, sendo extensamente utilizada na literatura nos pacientes após AVE, de fácil utilização e abrangendo um grande número de domínios. A SS-QOL por ser uma escala específica, mostrou-se ser válida para acessar alterações significativas da qualidade de vida dentro das dimensões dos sintomas observados, desta forma, indicando ser uma escala mais direcionada as particularidades e domínios mais afetados pelos pacientes após AVE.

Entretanto, apesar de serem escalas reconhecidas internacionalmente, são necessários mais estudos clínicos aplicando-as nos indivíduos após AVE no Brasil, para melhor assegurar as suas propriedades psicométricas, aplicabilidade, meIhor forma de administração (auto-administrado ou entrevista) e as possíveis interferências interexaminadores.

10.Santos AS. Questionário específico de avaliação da qualidade de vida em pacientes portadores de doença cérebro vascular do tipo isquêmica: tradução e adaptação cultural para a língua portuguesa falada no Brasil (Dissertação). Florianópolis: Universidade Federal de Santa Catarina, 2000, 48p.

11.Suenkeler IH, Nowak M, Misselwitz B, Kugler C, Schreiber $\mathrm{W}$, Oertel $\mathrm{WH}$, et al. Timecourse of health-related quality of life as determined 3, 6 and 12 months after stroke: relationship to neurological deficit, disability and depression. J Neurol 2002;249:1160-7.

12.Bolsche $F$, Hasenbein $U$, Reissberg $H$, Schlote A, Wallesch CW. Results of in- vs out patient post-stroke rehabilitation over 6 months. Fortschr Neurol Psychiatr 2003;71:458-68.

13.Teixeira-Salmela LF, Faria CDCM, Guimaraes CQ, Goulart F, Parreira VF, Inacio EP, et al. Treinamento físico e destreinamento em hemiplégicos crônicos: impacto na qualidade de vida. Rev Bras Fisioter 2005;9:347-53.

14.Cordini KL, Oda EY, Furlanetto LM. Qualidade de vida de pacientes com história prévia de acidente vascular encefálico: observação de casos. J Bras Psiquiatr 2005;54:312-7.

15.Raimundo CRR. Avaliação da qualidade de vida após acidente vascular cerebral (Monografia). Brasília: Centro SARAH de Formação e Pesquisa, Rede Sarah de Hospitais de Reabilitação, 2002, 69p.

16.Constitution of the World Health Organization. In World Health Organization. Handbook of basic documents. 5 ed. Geneva: Palais des Nations, 1952, 3-20.

17.Lai SM, Perera S, Duncan PW, Bode R. Physical and social functioning after stroke: comparison of the Stroke Impact Scale and Short Form-36. Stroke 2003;34:488-93. 
18.Scipper H, Clinch JJ, Olweny CLM. Quality of life studies: definitions and conceptual issues. In: Spilker B. Quality of life and pharmacoeconomics in clinical trials. 2nd ed. Philadelphia: Lippincott-Raven, 1996, 11-23.

19.De Haan R, Aaronson N, Limburg M, Hewer RL, van Crevel H. Measuring quality of life in stoke. Stroke 1993;24(2):320-7. 20.Teixeira-Salmela LF, Magalhães LC, Souza AC, Lima MC, Lima RCM, Goulart F. Adaptação do perfil de saúde de Nottin gham: um instrumento simples de avaliação da qualidade de vida. Cad Saúde Pública 2004;20:905-14.

21.Kranciukaite $D$, Rastenyte $D$. Measurement of quality of life in stroke patients. Med (Kaunas) 2006;42:709-16.

22.Ciconelli RM, Ferraz MB, Santos W, Meinão I, Quaresma MR. Tradução para a língua portuguesa e validação do questionário genérico de avaliação de qualidade de vida SF-36 (Brasil SF-36). Rev Bras Reumatol 1999;39:143-50.

23.Williams LS, Weinberger M, Harris LE, Clark DO, Biller J. Development of a stroke-specific quality of life scale. Stroke 1999;30:1362-9.

24.Carod-Artal FJ. Escalas específicas para la evaluación de la calidad de vida en el ictus. Rev Neurol 2004;39:1052-62.

25.Hilari K, Owen S, Farrelly SJ. Proxy and self report agreement on the stroke and aphasia quality of life scale-39 (SAQOL-39). J Neurol Neurosurg Psychiatr 2007;78:1072-5.

26.Anderson C, Laubsher S, Burns R. Validation of the short form 36 (SF-36) health survey questionaire among stroke patients. Stroke 1996;27:1812-6.

27.Ware JE, Kosinski M, Keller SD. A 12-item short-form health survey: construction of scales and preliminary test of reliability and validity. Med Care 1996;34:220-6.
28.The World Health Organization. Quality of Life Assessment (WHOQOL): development and general psychometric properties. Soc Sci Med 1998;46:1569-85.

29.Fleck MPA, Louzada S, Xavier M, Chachamovich E, Vieira G, Santos L, et al. Desenvolvimento da versão em português do instrumento de avaliação de qualidade de vida da organização mundial da saúde (WHOQOL-100). Rev Bras Psiquiatr 1999;21:19-28.

30.Fatoye FO, Komolafe MA, Eegunranti BA, Adewuya AO, Mosaku SK, Fatoye GK. Cognitive impairment and quality of life among stroke survivors in Nigeria. Psychol Rep 2007;100:87682.

31.Ewert T, Stucki G. Validity of the SS-QOL in Germany and in survivors of hemorrhagic or ischemic stroke. Neurorehabil Neural Repair 2007;21:161-8.

32.Muus I, Williams LS, Ringsberg KC. Validation of the stroke specific quality of life scale (SS-QOL): test of reliability and validity of the Danish version (SS-QOL-DK). Clin Rehabil 2007;21:620-7.

33.Angeleri F, Angeleri VA, Foschi N, Giaquinto S, Nolfe G. The influence of depression, social activity, and family stress on functional outcome after stroke. Stroke 1993;24:1478-83.

34.Duncan PW, Samsa GP, Weinberger M, Goldstein LB, Bonito A, Witter DM, et al. Health status of individuals with mild stroke. Stroke 1997;28:740-5.

35.Astrom M, Asplund K, Astrom T. Psychosocial function and life satisfaction after stroke. Stroke 1992;23(4):527-31. 